\title{
Prévalence du dessèchement du manguier et evaluation de la fréquence des champignons associés à la maladie au Burkina Faso.
}

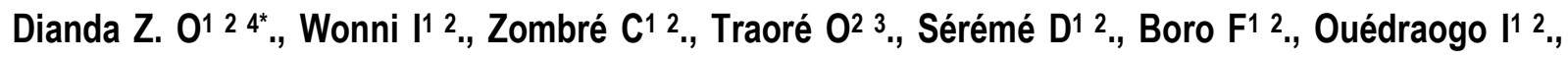
Ouédraogo S. L ${ }^{12}$., Sankara P4

${ }^{124}$ Ouamarou Z. Dianda, Institut de l'Environnement et de Recherches Agricoles (INERA)/Centre National de Recherche Scientifique et Technologique (CNRST) , Station de Farako-Ba, 01 BP 910 Bobo Dioulasso 01, Burkina-Faso, oumarou.dianda@yahoo.fr

12 Dr. Issa Wonni, Institut de l'Environnement et de Recherches Agricoles (INERA)/ Centre National de Recherche Scientifique et Technologique (CNRST), Station de Farako-Ba, 01BP 910 Bobo Dioulasso 01, Burkina-Faso, wonniissa@yahoo.fr.

1 2Dr. Cyrille Tinlé Zombré, Institut de l'Environnement et de Recherches Agricoles (INERA)/ Centre National de Recherche Scientifique et Technologique (CNRST), Station de Farako-Ba, 01BP 910 Bobo Dioulasso 01, Burkina-Faso, cyrille.zombre@yahoo.fr.

2 30umarou Traoré, Institut de Recherche en Sciences Appliquées et Technologiques (IRSAT)/ Centre National de Recherche Scientifique et Technologique (CNRST); 01BP 2393 Bobo-Dioulasso 01 ; Burkina Faso, oumaroutraor@yahoo.fr.

12Dr Idrissa Sérémé Institut de l'Environnement et de Recherches Agricoles (INERA)/ Centre National de Recherche Scientifique et Technologique (CNRST), Station de Kamboenssin 04 BP8645 Ouagadougou 04 sereme@gmail.com. 12 Fousseni Borro Institut de l'Environnement et de Recherches Agricoles (INERA), Station de Farako-Ba, 01 BP 910 Bobo Dioulasso 01, Burkina-Faso fousseniboro@yahoo.fr.

1 2Dr. Léonard Ouédraogo, Institut de l'Environnement et de Recherches Agricoles (INERA)/ Centre National de Recherche Scientifique et Technologique (CNRST), Station de Farako-Ba, 01 BP 910 Bobo Dioulasso 01, Burkina-Faso, tinkoglega@gmail.com.

$12 \mathrm{Dr}$ Ibrahima Ouédraogo Institut de l'Environnement et de Recherches Agricoles (INERA)/ Centre National de Recherche Scientifique et Technologique (CNRST), Station de Farako-Ba, 01 BP 910 Bobo Dioulasso 01, Burkina-Faso oueibramina@yahoo.fr.

4Pr. Philippe Sankara, Université Ouaga I Pr Joseph KI-ZERBO, École doctorale Science et Technologie, Ouagadougou, Burkina Faso, philippe.sankara@ird.fr

*Auteur pour la correspondance : DIANDA Z. Oumarou Tel : 00226 73690211/75333401 Email : oumarou.dianda@yahoo.fr.

Original submitted in on $5^{\text {th }}$ April 2018. Published online at www.m.elewa.org on $30^{\text {th }}$ June 2018 https://dx.doi.org/10.4314/jab.v126i1.6

\section{RÉSUMÉ}

Prévalence du dessèchement du manguier et evaluation de la fréquence des champignons associés à la maladie au Burkina Faso.

Objectif: l'objectif de l'étude est de contribuer au diagnostic du dessèchement du manguier au Burkina Faso. II s'agit d'une part d'évaluer l'incidence et la sévérité de la maladie sur cinq variétés commerciales provenant du 
Nahouri, de la Sissili, du Houet, de la Comoé, du Kénédougou et de la Léraba et d'autre part d'inventorier les espèces de champignons associées aux symptômes de la maladie.

Méthodologie et résultats : Une échelle de 1 à 5 a été utilisée pour l'évaluation de l'incidence et de la sévérité du desséchement du manguier dans six provinces du Burkina Faso. Sa prévalence a été évaluée sur cinq variétés commerciales du pays. Les taux d'infections des espèces de champignons ont été calculés à partir des échantillons des manguiers malades. Les résultats révèlent une forte incidence du dessèchement de manguier dans le Nahouri ( $86 \%$ ) et une forte sévérité $(58,2 \%)$. Cependant, les plus faibles valeurs ont été constatées dans la Léraba avec $42,8 \%$ d'incidence et $29,8 \%$ de sévérité. Le Kénédougou, le Houet, la Comoé et la Sissili ont enregistré des incidences moyennes comprises entre $(43,6 \%)$ à $(81,6 \%)$ et de sévérités moyennes entre $(30,88 \%)$ à $(50,8 \%)$. La plus forte prévalence $(77 \%)$ a été enregistrée sur la variété Amélie, suivie de Kent, Keitt, et Lippens. Le plus faible taux (34\%) a été observé sur Brooks. Sept espèces de champignons ont été inventoriées. Le plus fort taux d'infection a été enregistré pour le champignon Pestalotiopsis microspora (53,5 $\%$ ), suivi de Botryodiplodia theobromae, Fusarium sp, Curvularia sp, Aspergillus niger, et de Botrytis cinerea. Le plus faible taux (1.25\%) a été noté pour Colletotrichum gloesporiodes.

Conclusion et Application: Ces résultats renseignent de l'importance du dessèchement des manguiers ainsi que les espèces de champignons associées à la maladie au Burkina Faso. Des études de caractérisation moléculaire couplées au test de pathogénie devraient permettre d'incriminer les espèces fongiques responsables de la maladie pour mieux la contrôler.

Mots clés : Dessèchement, Manguier, Incidence, Sévérité, Champignons, Burkina Faso.

\section{ABSTRACT \\ Prevalence of mango tree decline and evaluation of fungi frequency associated disease in Burkina Faso}

Objective : The objective of the study is to contribute to the diagnosis of the mango tree decline in Burkina Faso. It is to evaluate incidence and severity of this disease in five commercial varieties from Nahouri, Sissili, Houet, Comoé, Kénédougou and Léraba and to inventory the fungal species associated with the symptoms of the disease.

Methodology and results : Incidence and severity of mango tree decline symptoms were calculated according to allotted scale from 1 to 5 on diseased trees. Prevalence was also assessed on Kent, Keitt, Amélie Lippens and Brooks commercial varieties from the six previously mentioned producing provinces of Burkina Faso. Samples of infected plant were collected and incubated for the inventory and infection rate of the fungal species. The frequency of fungal species were also carried out from the different organs of the mango tree. Survey results showed highest mango decline incidence $(86 \%)$ and severity $(58.2 \%)$ in the visited orchards of Nahouri. However, least incidence of (42.8\%) and severity of (29.8\%) were observed in Léraba. Intermediate values of incidence and severity of the desease were observed in Kénédougou, Houet and Comoé. Maximum prevalence of disease $(77 \%)$ was calculated on cultivar Amélie followed by Kent ; Keitt, and Lippens. The minimum disease incidence (34\%) was noted on Brooks. Seven species of fungi were inventoried. The highest frequency was recorded in Pestalotiopsis microspora (53.5\%) followed by Botryodiplodia theobromae, Fusarium sp, Curvularia $\mathrm{sp}$, Aspergillus sp and Botrytis cinerea. The least rate (1.25\%) were noted in Colletotrichum gloesporiodes. Conclusion and application: These results indicate the importance of the mango decline and the species of fungi associated with the disease in Burkina Faso. Pathogenicity and molecular analyzes should allow to incriminate the fungal species responsible for the disease to better control.

Keys : decline, mango, incidence, severity, fungi, Burkina Faso

\section{INTRODUCTION}

Le manguier (Mangifera indica L.) est un important arbre fruitier des régions tropicales et subtropicales du monde. Au Burkina Faso la filière mangue joue un rôle socio-economique important. Elle occupe 

des champignons associés à la maladie au Burkina Faso.

près de 15000 producteurs et la production de mangue est estimée à 33701 ha soit $58 \%$ de la superficie du verger national (PAFASP, 2011). Elle constitue la prémière culture fruitière du pays et a connu une importante évolution ces dernières années passant ainsi de 160000 tonnes en 2009 à plus de 404000 tonnes en 2014 (Bakouan, 2015). En outre la transformation occupe une place importante dans la filière mangue au Burkina Faso. En 2016, on a enrégistré environ 1930 tonnes de mangues séchées et 2335 tonnes de mangues transformées en jus et purée. L'exportation de la mangue et ses produits dérivés procure d'importantes devises aux différents acteurs. En effet, en 2016, le pays a exporté 8481,589 Tonnes de mangues fraiches et 1755,6 Tonnes de mangues séchées. En termes de ressources, la filière mangue a généré un revenu de plus de 33156348 USD (APROMAB, 2016). Les principales zones de production sont rencontrées dans les régions des Hauts Bassins, des Cascades, du Centre-Ouest et du Sud-Ouest. Les variétés Kent, Keitt, Amélie, Lippens et Brooks figurent parmi les plus cultivées à cause de leur rendement à l'hectare, leur excellent goût, leur valeur hautement nutritive, et surtout leur valeur commerciale (APROMAB, 2016). En dépit de ces atouts, la production et l'exportation des mangues au Burkina Faso sont confrontées à de nombreux problèmes phytosanitaires. La maladie des taches noires du manguier causée par Xanthomonas citri pv. mangiferaeindicae et l'anthracnose du manguier causée par Colletotrichum gloesporiodes constituent les principales maladies rencontrées dans les zones de production (Pruvost et al., 2011 ; Zombré, 2016). Parmi les maladies figure le dessèchement $d u$ manguier, encore appélé syndrome de mort subite ou mortalité des plantes du manguier (Khaskheli et al., 2011). Cette maladie est considérée comme l'une des menaces les plus sérieuses dans toutes les régions productrices de mangues (Kazmi et al., 2005). En effet cette maladie a été signalée en Egypte, en Australie, aux Emira Arabes Unies et au Pérou (Sakalidis et al., 2011; Ismail et al., 2012; Saeed et al., 2017; Rodríguez et al., 2017). Elle a été également décrite au Brésil, en Oman et au Pakistan (Oliveira et al., 2015). D'après des études prospectives, de nombreux pays producteurs dont le Burkina Faso seraient propices au développement de cette maladie (Galdino et al., 2016). Au Burkina Faso, le dessèchement du manguier existait depuis fort longtemps mais les dégâts étaient mineurs. Cependant, en 2015 des cas de fortes mortalités de manguiers occasionnés par le dessèchement ont été observés dans certaines zones de production sur les variétés et accessions cultivées de différents âges. Le dépérissement qui se traduit par les flétrissements des feuilles évoluant progressivement vers les dessèchements des rameaux et des branches avec ou sans défoliation, les gommoses à travers l'apparition des exsudats brunâtres sur les troncs et branches, les brunissements des troncs et des branches, les craquélures des écorces, et les pourritures racinaires constituent les diverses manifestations pouvant conduire au dessèchement du manguier (Ploetz et al., 1996 ; Masood et al., 2010; Ismail., 2011; Naqvi et al., 2014). Ces symptômes ont été retrouvés seuls ou en combinaison de deux ou de plusieurs dans différents vergers de mangue au Brésil et au Pakistan. Le dessèchement affecte la vitalité et le rendement du manguier et devient une importante ménace pour l'utilisation industrielle de la mangue au niveau mondiale (Anwar et al., 2012 ; Suresh., 2014). En effet, cette maladie peut provoquer la mort du manguier, deux mois environ après les toutes premières infections. Les différents stades de croissance, de la pépinière jusqu'aux plantations sont concernés (Oliveira et al., 2015). Au Pakistan les pertes liées à cette maladie varient entre 20 à $60 \%$ en fonction des zones de production (Masood et al., 2012). AlAdawi et al., (2003) ont signalé le dessèchement en tant que nouvelle maladie du manguier affectant $60 \%$ des arbres dans la région d'Al Batinah en Oman. En 2005, une étude rapportée par I'Institut National de la Recherche Agronomique du Niger (INRAN) a constaté que la région de Gaya était la plus touchée par le dépérissement $d u$ manguier avec $80 \%$ des pieds attaqués et $35 \%$ de mortalité (Adamou., 2005). L'évaluation de la prévalence du dessèchement à partir des observations au champ sur certains cultivars de manguiers au Pakistan a révèlé que toutes les variétés sont attaquées avec des taux variables 
(Rehman et al., 2011; Naqvi et Perveen., 2015). L'agent causal de cette maladie est resté pendant de nombreuses années difficile à cerner en raison des différents champignons qui lui sont associés (Ploetz., 2003). En effet, Lasiodiplodia theobromae, Neofusicoccum ribis, Botryosphaeria dothidea, Diplodia sp, Pseudofusicoccum sp, Ceratocystis sp, Fusarium sp et Nattrassia mangiferae sont des espèces de champignons signalées comme responsables de cette maladie. Elles peuvent individuellement ou en combinaison infecter les manguiers et provoquer le dessèchement (Sakalidis et al., 2011; Fateh et al., 2016). Des contraintes édaphiques (pauvrété, $\mathrm{pH}$, structure et texture des sols) des facteurs climatiques (fortes températures,

\section{MATÉRIEL ET MÉTHODES}

Zone d'étude : Les prospections ont été réalisées d'Avril à Août 2016 et 2017. La figure1 illustre les sites prospectés dans les six provinces répresentant des zones de production de mangues au Burkina Faso. En effet, le Kénédougou, la Comoé, la Léraba et le Houet contribuent à $75 \%$ de la production fruitière nationale (Guira, 2003). Ces quatre provinces sont situées dans la zone sudsoudanienne. Cette zone agroécologique a une végétation riche composée de savanes arborées, arbustives et boisées, et de forêts claires. Elle est à faible pluviométrie) et certaines pratiques agricoles inadéquates (fertilisations non recommandées, mauvaise irrigation...) peuvent prédisposer ou favoriser le développement de la maladie (Naqvi et al., 2015 ; Galdino et al., 2016 ; Hassan et al., 2017). Depuis l'explosion du dessèchement de manguier au Burkina Faso, aucune étude d'inventaire, d'évaluation des pertes et de caractérisation des agents fongiques associés à la maladie n'a été réalisée. Cette étude a pour objectif d'évaluer l'incidence et la sévérité du dessèchement du manguier sur les variétés commerciales, et d'inventorier les espèces de champignons associées au dessèchement dans les grandes zones de production.

vocation agricole caractérisée par les cultures pérennes (Manguiers, agrumes, anacardiers et jatropha) et les cultures annuelles (Coton, igname, sorgho, mil et maïs). La pluviométrie annuelle se situe entre $900 \mathrm{~mm}$ et 1200 $\mathrm{mm}$. En revanche, le Nahouri est situé dans la zone nordsoudanienne présentant une pluviométrie comprise entre $700 \mathrm{~mm}$ à $900 \mathrm{~m}$ (Bonfigliol, 2010). D'une manière générale, la province présente des sols du type ferrugineux tropical peu favorable aux activités agricoles (Felix., 1989).

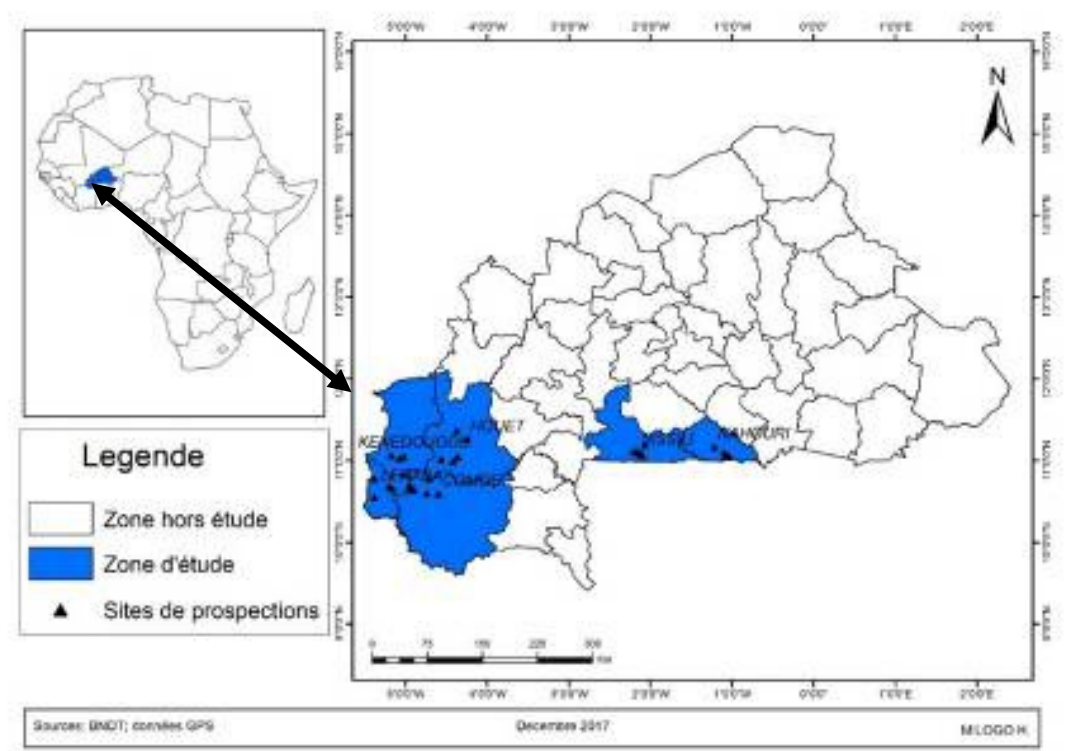

Figure 1: Carte répresentant les différentes zones d'étude. 

des champignons associés à la maladie au Burkina Faso.

Evaluation de l'incidence et de la sévérité : L'étude a consisté à choisir de façon aléatoire cinq vergers variant d'au moins 1 à 2 ha dans cinq localités distantes d'au moins $5 \mathrm{~km}$ dans chaque province. L'évaluation de l'incidence et de la sévérité a été effectuée sur 50 arbres choisis aléatoirement dans chaque plantation suivant les diagonales. Les échelles de Ramos et al., (1997) et de Masood et al., (2010) ont été utilisées pour évaluer la sévérité. L'évaluation a été faite à travers des observations visuelles sur les côtés Nord, Sud, Est et Ouest de la couronne de chaque arbre.

Tableau 1 : Échelles de Ramos et al., (1997); et Masood et al., (2010) utilisées pour l'evaluation de l'incidence et de la sévérité du dessèchement du manguier.

\begin{tabular}{c|c|l}
\hline Degré d'attaque & $(\%)$ de la canopée atteinte & $\begin{array}{l}\text { Caractéristiques des symptômes } \\
\text { Plantes apparemment saines, absence des feuilles } \\
\text { nécrosées. }\end{array}$ \\
\hline 1 & 25 & $\begin{array}{l}\text { Stade précoce de l'infection caractérisé par la présence des } \\
\text { brunissements sur les pétioles et des nécroses briques } \\
\text { marginales sur les limbes des feuilles sur quelques } \\
\text { branches. }\end{array}$ \\
\hline 3 & 50 & $\begin{array}{l}\text { Présence de feuilles desséchées avec ou sans défoliation } \\
\text { sur environ la moitié des branches de l'arbre. }\end{array}$ \\
\hline 4 & 75 & $\begin{array}{l}\text { Feuilles desséchées sur le maximum de branches avec ou } \\
\text { sans défoliation progressives. }\end{array}$ \\
\hline 5 & 100 & $\begin{array}{l}\text { Cas sévère, infection généralisée sur toute la canopée, } \\
\text { mort de l'arbre. }\end{array}$ \\
\hline
\end{tabular}

Le taux d'incidence du dessèchement a été estimé à partir du rapport entre le nombre d'arbres malades et le nombre total d'arbres inspectés (Cooke., 2006). $I=\sum\left(\frac{n}{N}\right) x 100 \quad I$ : incidence moyenne par province $n$ : nombre de plantes malades par localité de chaque province $N$ : nombre total de plants observés dans une province donnée ( $N=250)$.

L'indice de sévérité de la maladie sur un site a été déterminé à l'aide de l'équation suivante (Kranz., 1988): avec $I s=\sum\left(\frac{X i x n i}{N x Z}\right) x 100$ avec : Is: Indice de sévérité de la maladie sur le site, $X i$ : Sévérité $i$ de la maladie sur l'arbre, $n i$ : Nombre d'arbres de sévérité $i$, $N$ : Nombre total d'arbres observés $(N=50)$ et $Z$ : Échelle de sévérité la plus élevée la note 5 .

L'incidence du dessèchement du manguier en fonction de cinq variétés commerciales : Cette étude a été réalisée sur cinq variétés commerciales à savoir : Kent, Keitt, Amélie, Brooks et Lippens. Elles sont produites dans toutes les provinces exceptées le Nahouri. Vingt arbres de chaque variété ont été retenus de façon aléatoire par province pour l'étude. L'incidence du dessèchement sur une variété $i$, dans une province correspond : $P i=\frac{(X i x 100)}{N i} P i$ : incidence de la maladie sur une variété dans une province, $X i$ : nombre de plantes malades sur la variété $i$. Ni: Nombre total de plantes observées sur la variété $i$. Ainsi l'incidence moyenne de la maladie sur une variété donnée est obtenue à partir de celles des cinq provinces concernées. Échantillonnage des organes infectés : L'échantillonnage a été réalisé dans les bassins de production de mangues au Burkina Faso dont les provinces du Kénédougou, du Houet, de la Comoé et de la Léraba. Dans chaque province des échantillons de feuilles, de rameaux et de racines ont été collectés sur 100 manguiers malades povenant des cinq localités. Au sein de chaque verger le prélèvement a été réalisé sur vingt pieds choisis de façon aléatoire suivant les diagonales. Une collecte spontanée des organes malades a été réalisée dans le Nahouri et la Sissili. Les échantillons ont été placés dans des enveloppes ou des sachets plastiques comportant le numéro de collecte, la date, le nom et les coordonnées géographiques de la localité. Ils sont transportés et conservés à $4^{\circ} \mathrm{C}$ au laboratoire pour l'étude de l'inventaire et le taux d'infection des différentes espèces.

Inventaire et le taux d'infection des espèces de champignon associés à la maladie : Des morceaux des différents organes collectés ont été désinfectés 
respectivement dans de l'alcool $70 \%$, l'eau de javel $1 \%$ puis dans l'eau distillé stérile. Ils sont placés dans des boites de Pétri contenant des papiers buvards précédemment stérilisés et humidifiés à l'eau distillé stérile. Chaque boite contient quatre fragments de chaque type d'organe. Les différents échantillons ont été mis en incubation à $28^{\circ} \mathrm{C}$ sous une alternance de 12 heures à la lumière UV et 12 heures à l'obscurité pendant 7 jours. Les conidies et des spores observées sur les différents organes à la loupe binoculaire et au microscope ont été comparées avec les données déjà existantes dans la clé d'identification de Mathur et Kongsdal, (2003) et les récherches bibliographiques. Ces caractéristiques morphologiques ont été utilisées pour l'inventaire des différentes espèces de champignon.

Taux d'infection des espèces de champignons : Les analyses microbiologiques réalisées selon la méthode de Larignon et Dubos, (1997) ont été conduites sur 400 manguiers provenant des 4 provinces concernées par l'étude. Un manguier infecté est caractérisé par la présence de l'espèce de champignon sur au moins un des trois types de ses organes. Ainsi, le taux d'infection

\section{RÉSULTATS}

Symptômatologie du dessèchement du manguier au Burkina Faso : Le dépérissement, la gommose, et les pourritures racinaires peuvent conduire au dessèchement du manguier. Au Burkina Faso, des symptômes similaires à la maladie ont été rencontrés. II s'agit notamment des : Symptômes du dépérissement: lls ont été les plus fréquemment observés. Ils se traduisent par des flétrissements de feuilles notamment un jaunissement d'une des espèces inventoriées dans une province correspond au nombre d'arbres infectés sur le nombre total de plantes observées. II se calcule à travers la formule suivante : $T i=\frac{n i x 100}{N}$.

$T i$ : Taux d'infection d'une espèce de champignon donnée $n i:$ nombre des arbres infectés par l'espèce et $N$ : nombre total des arbres concernés par l'étude. Le taux d'infection moyenne de chaque espèce a été calculé à partir de ceux provenant des quatre provinces.

Analyses statistiques: Le tableur excel 2013 a été utilisé pour la saisie et l'organisation des données ainsi que la réalisation des graphiques. Les données d'incidences et de sévérités dans les différentes localités, ainsi que la prévalence sur les différentes variétés provenant des quatre provinces ont été soumises à des analyses de variances (ANOVA) à l'aide du logiciel XLSTAT 2007. La séparation des moyennes a été faite par le test de Newmans Keuls au seuil de $5 \%$.

suivi de l'apparition des nécroses rouges briques marginales ou terminales sur les limbes (Figure $2 \mathrm{~A}$ ). Ils aboutissent au dessèchement de toute la surface foliaire pouvant progresser jusqu'aux rameaux (Figure $2 \mathrm{~B}$ ). A partir d'un rameau atteint la maladie peut progresser sur une branche puis les autres et entrainer un dessèchement partiel (Figure $2 \mathrm{C}$ ) ou total avec ou sans défoliation (Figure 2D). 

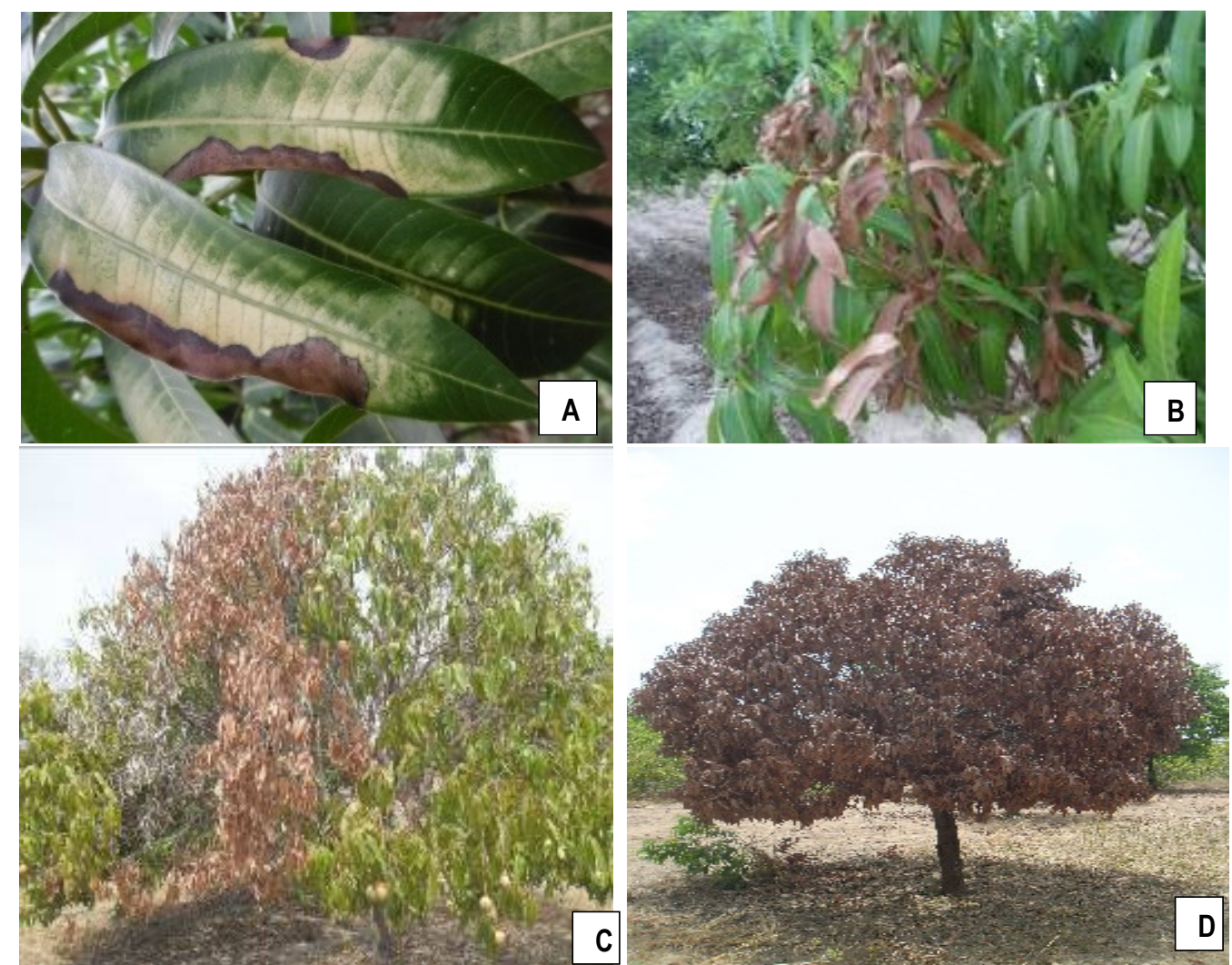

Figure 2 : Symptômes du dépérissement $(A)$ : Jaunissement et apparition des nécroses rouges briques marginales $(B)$ : Dessèchement des feuilles et des rameaux, (C) : Dessèchement partiel de l'arbre, (D) : Dessèchement total avec les feuilles toujours attachées aux branches.

Autres symptômes du dessèchement du manguier : Ils ont été occasionnellement observés dans certains vergers en association avec les symptômes du dépérissement. II s'agit : des exsudats brunâtres sur des troncs et des branches (Figure $3 \mathrm{~A}$ et $\mathrm{B}$ ) rencontrés dans les vergers de Nahouri. Dans le Houet, des coupes transversales et longitudinales sur des branches et troncs des manguiers sévèrement attaqués montrent des noircissements et des brunissements vasculaires (Figure $3 \mathrm{~B}$ et $\mathrm{C}$ ). En outre des perforations sur des troncs ont été également observées. Des investigations menées sporadiquement au niveau racinaire dans les vergers de la même localité ont permis de constater des pourritures avec des odeurs nauséabondes.
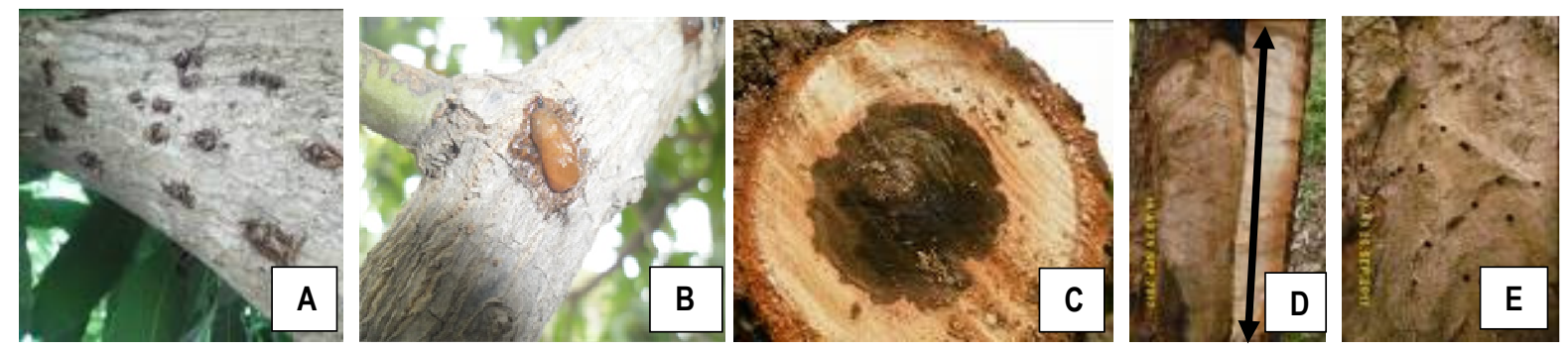

Figure 3 : Symptômes occasionnellement observés sur les manguiers malades : (A et B): Exsudats gommeux brunâtre sur les branches des arbres; (C) : Coupe transversale montrant un noircissement vasculaire du tronc du manguier ; (D) : Coupe longitidinale illustrant une partie infectée et une partie saine $(E)$ : Perforations sur les ecorces du tronc similaires aux trous provoqués par des insectes vecteurs des champignons responsables de la maladie. 
Incidence du dessèchement du manguier au Burkina

Faso : Les plus fortes valeurs de l'incidence proviennent du Nahouri $(86 \%)$ et de la Sissili $(81,6 \%)$. Les taux intermédiaires ont été enregistrés dans le Houet $(60,4 \%)$ et dans la Comoé $(62,8 \%)$. En révanche, les plus faibles incidences moyennes ont été observées dans la Léraba $(42,8 \%)$ et dans le Kénédougou (43,6\%) (Figure 4).

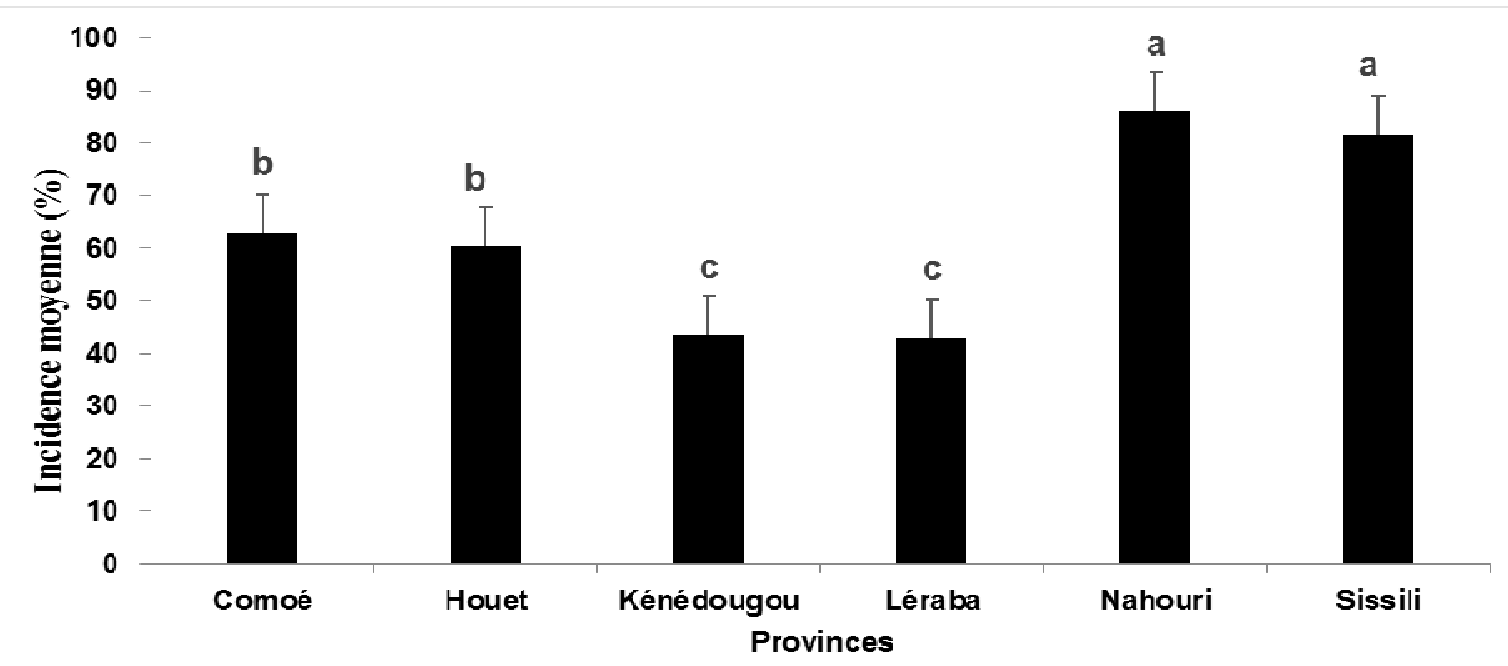

Figure 4: Incidences moyennes du dessèchement du manguier dans cinq provinces du Burkina Faso. Les valeurs suivies d'une même lettre ne sont pas significativement différentes au seuil de $5 \%$ selon le test de Newman keuls.

Sévérité du dessèchement du manguier au Burkina Faso: Tout comme l'incidence, la plus forte valeur de séverité moyenne a été également observée dans le Nahouri avec $58,2 \%$. Elle est suivie de la Sissili avec $50,8 \%$ de sévérité. Par ailleurs de nombreux cas de mortalités ont été enregistrés non seulement dans ces deux provinces mais également dans les autres excepté la Léraba. La plus faible sévérité moyenne a été observée dans la Léraba $(29,84 \%)$ et est suivie de celle du Kénédougou $(30,88 \%)$. Les sévérités moyennes intermédiaires proviennent du Houet avec $37,2 \%$ et de la Comoé avec 38,48\% (Figure 5).

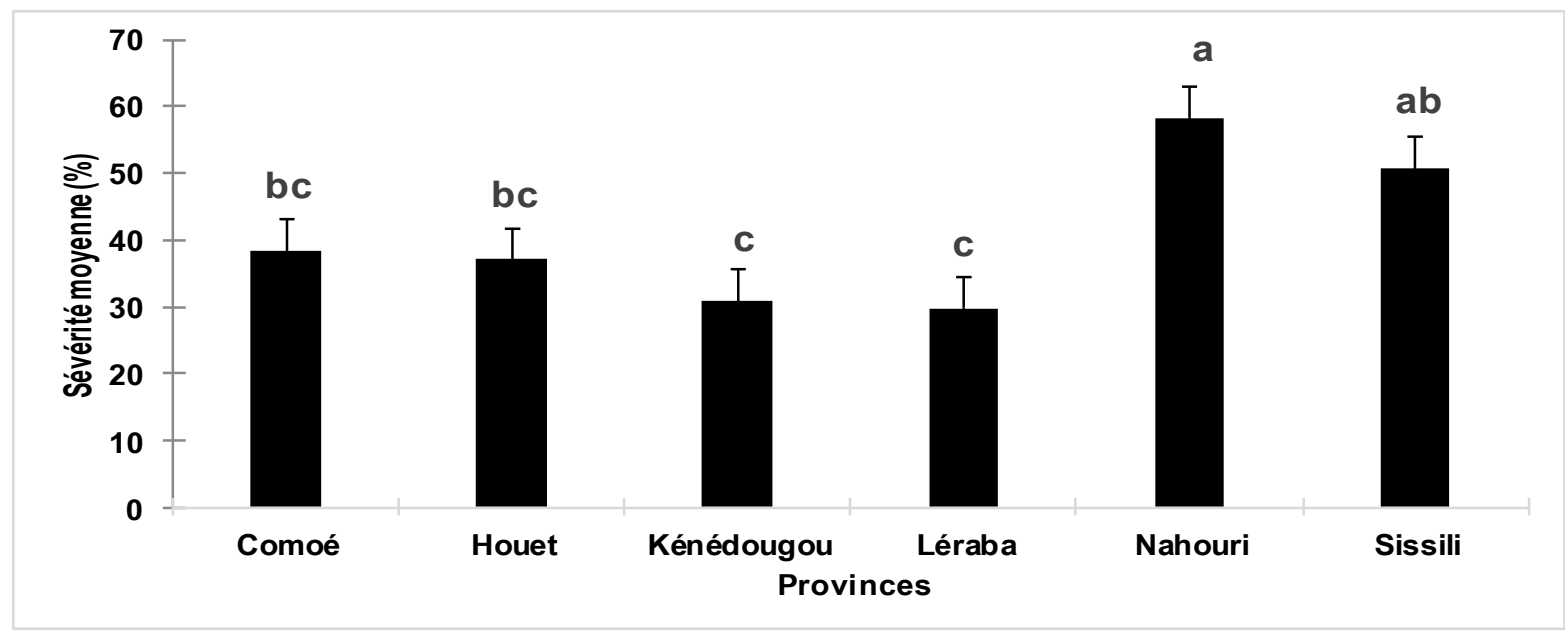

Figure 5: Sévérité moyenne du dessèchement du manguier dans six provinces du Burkina Faso. Les valeurs suivies d'une même lettre ne sont pas significativement différentes au seuil de $5 \%$ selon le test de Newman keuls. 
Prévalence du dessèchement du manguier sur les principales variétés commerciales : Les prospections ont permis de constater la présence du dessèchement du manguier sur toutes les variétés et accessions. Cependant, l'incidence moyenne varie selon les variétés.
Ainsi les plus fortes valeurs de l'incidence ont été enrégistrées sur Amélie (77\%), Kent (61\%) et Keitt $(56 \%)$; les plus faibles incidences moyennes ont été observées sur les variétés Brooks et Lippens avec respectivement $34 \%$ et $42 \%$ d'incidence (Figure 6 ).

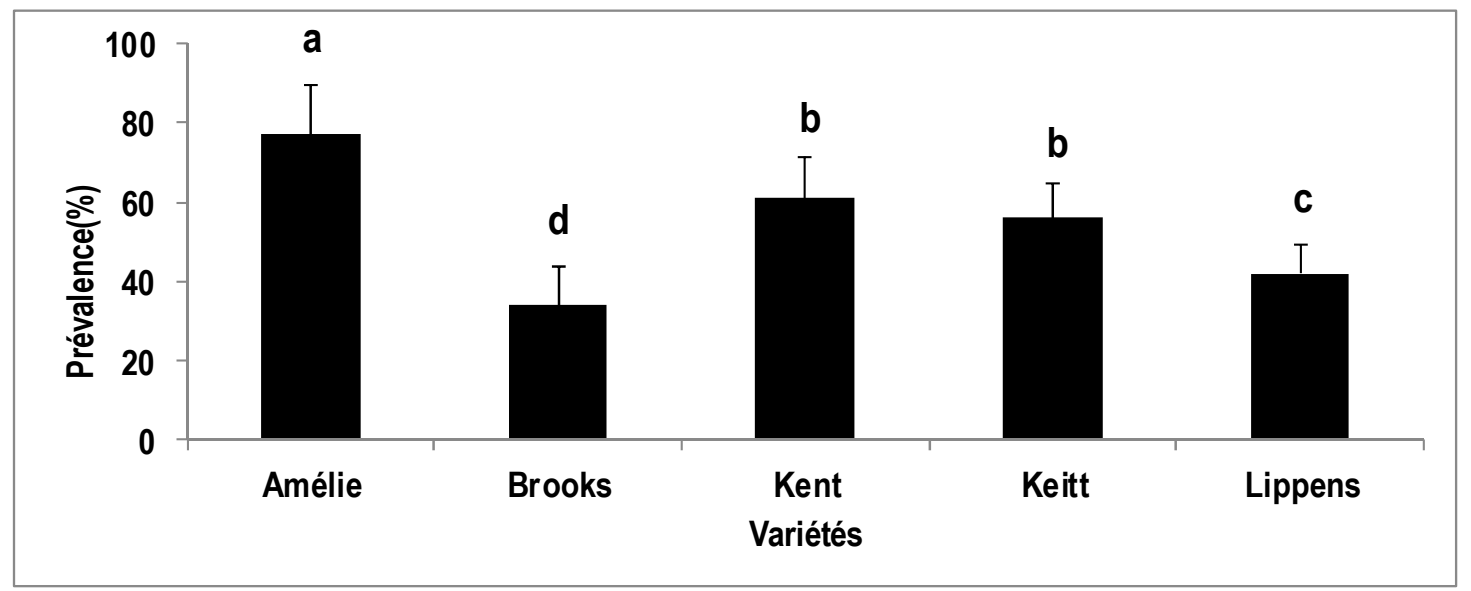

Figure 6: Incidence moyenne du dessèchement en fonction de cinq variétés commerciales du Burkina Faso. Les valeurs suivies d'une même lettre ne sont pas significativement différentes au seuil de $5 \%$ selon le test de Newman keul.

Inventaire des espèces de champignons associées au dessèchement du manguier : A partir des échantillons du dessèchement du manguier, sept espèces de champignons ont été inventoriées. Elles ont été détectées sur la base des caractéristiques morphologiques sur

milieu de cultures PDA et au microscope. Elles se repartissent en deux groupes:

Les espèces fréquemment observées : Trois espèces ont été rencontrées sur tous les organes collectés provenant de toutes les provinces. II s'agit de : Pestalotiopsis microspora, Botryodiplodia theobromae et Fusarium sp (Figure 7).
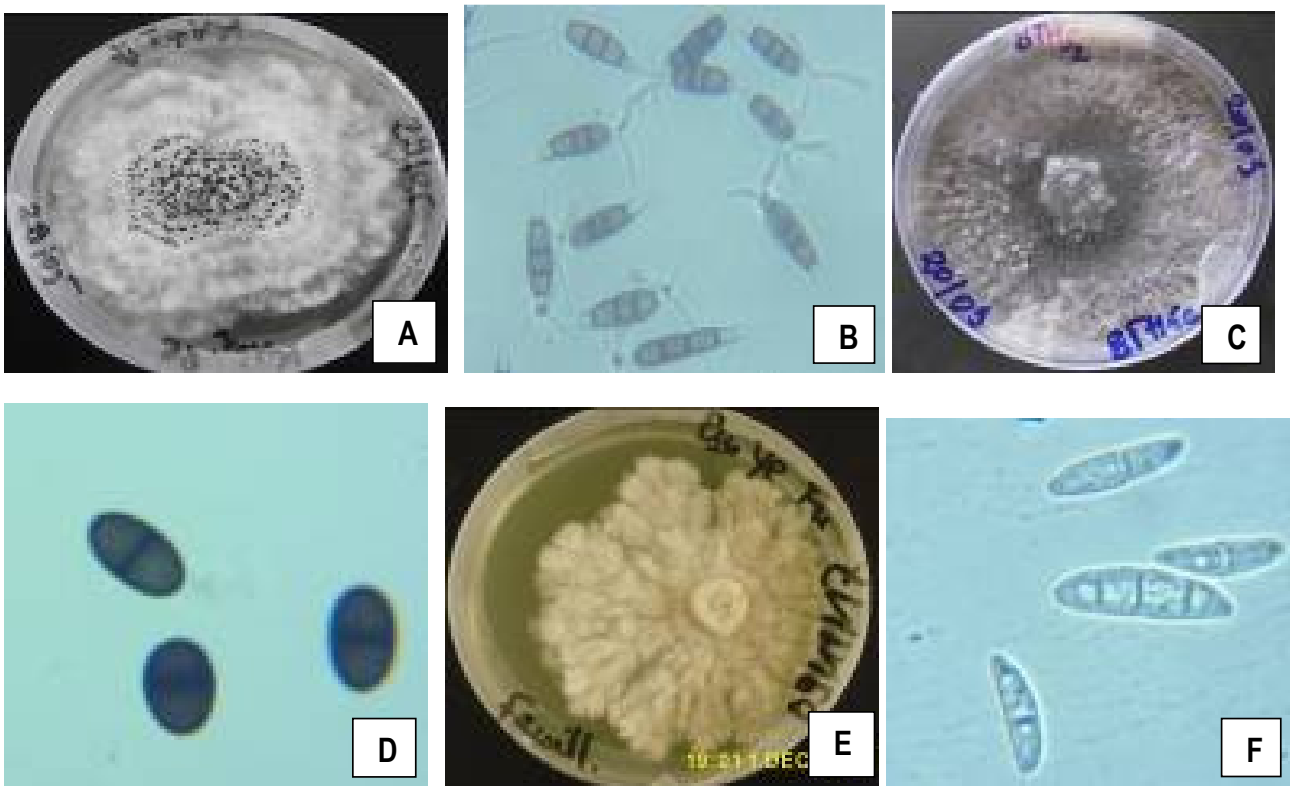

Figure 7 : Espèces de champignon fréquement observées à partir des différents organes symptômatiques: ( A et B ): P. microscopora, ( $C$ et $D$ ): B. theobromae , ( $E$ et $F$ ): Fusarium sp. 

des champignons associés à la maladie au Burkina Faso.

Les espèces de champignons occasionnellement rencontrées : Elles n'ont pas été observées sur les racines. En outre, elles sont rarement présentes sur les autres types d'organe (feuilles ou rameaux) ainsi que dans les sites de collectes. II s'agit de : Colletotrichum gloesporiodes, Curvalaria sp, Botritis cinerae, Aspergillus niger (Figure 8).
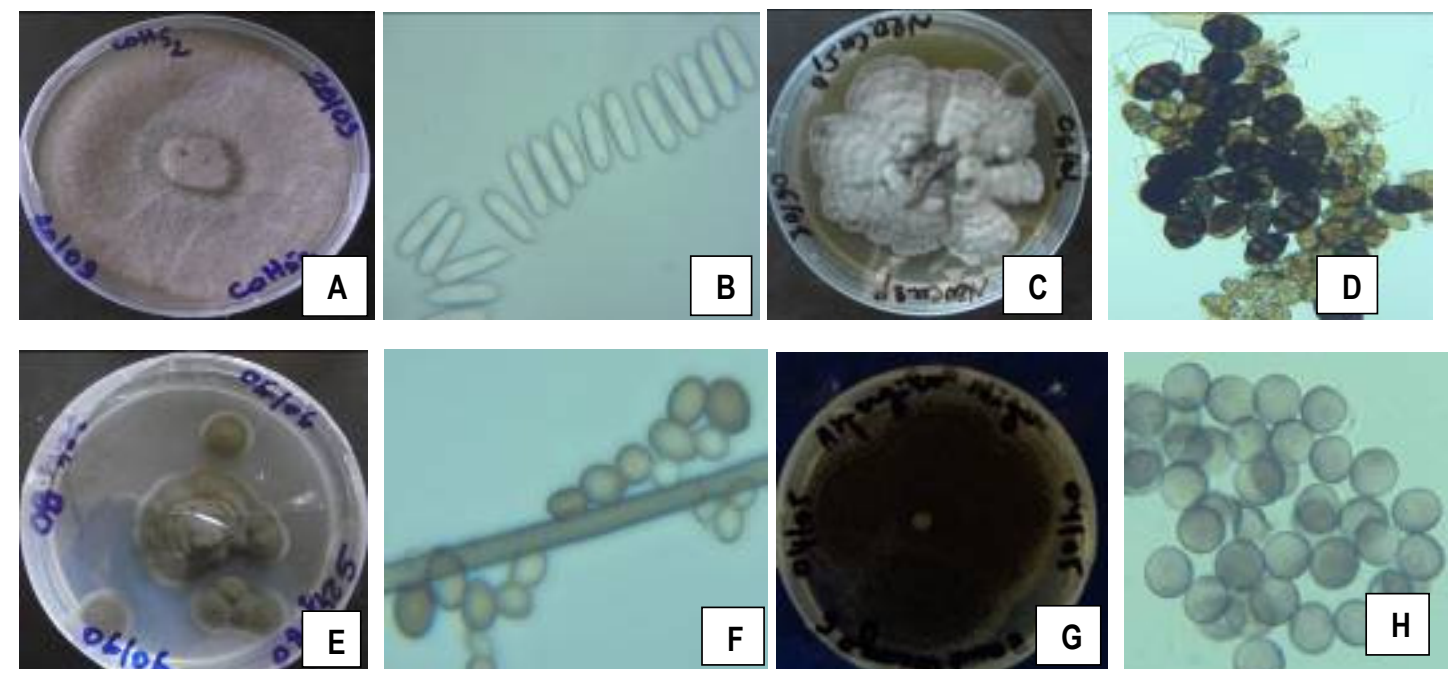

Figure 8 : Espèces de champignon occasionnellement observées sur les échantillons symptômatiques ( $A$ et $\mathrm{B}$ ): C. gloesporiodes, ( $\mathrm{C}$ et $\mathrm{D})$ : Curvularia sp, ( $\mathrm{E}$ et $\mathrm{F}$ ): B. cinerae, $(\mathrm{G}$ et $\mathrm{H})$ : A. niger.

Fréquence des espèces de champignons associées au dessèchement du manguier: Les différentes espèces de champignons précédemment citées ont été classées en deux catégories en fonction de leur fréquence. D'une part, on a les espèces fréquemment observées sur les feuilles, les rameaux et les racines (tableau 2) et présentent dans toutes les provinces. En effet, ces espèces ont enrégistré les plus fort taux d'infection, il s'agit de : P. microspora (53,5\%), B . theobromae (35,5\%), et Fusarium sp ( 6,5\% ). D'autre part, on note les espèces occasionnellement rencontrées. Les plus faibles taux d'infection proviennent de celles-ci. II s'agit de C. gloesporiodes (1,25\%), A.niger ( 2,25\%), B. cinerae (2,5\%), et Curvularia sp ( $5,75 \%)$.

Tableau 2: Le taux d'infection des différentes espèces de champignons en fonction des différentes provinces.

\begin{tabular}{llllllll} 
& $\boldsymbol{P}$. & $\mathbf{B}$ & Fusarium & Curvularia & $\mathbf{C}$ & $\boldsymbol{B}$. & $\boldsymbol{A}$ \\
microspora & theobromae & sp & sp & .gloesporiodes & cinerae & niger \\
\hline Comoé & 52 & 38 & 13 & 18 & 2 & 2 & 2 \\
\hline Houet & 58 & 42 & 9 & 0 & 1 & 3 & 1 \\
\hline Kénédougou & 64 & 33 & 2 & 3 & 0 & 0 & 3 \\
\hline Léraba & 40 & 29 & 2 & 2 & 2 & 5 & 3 \\
\hline $\begin{array}{l}\text { T(\%) } \\
\text { moyenne }\end{array}$ & 53,5 & 35,5 & 6,5 & 5,75 & 1,25 & 2,5 & 2,25 \\
\hline
\end{tabular}

\section{DISCUSSION}

Symtômatologie du dessèchement au Burkina Faso : Le dessèchement du manguier est une maladie complexe susceptible d'infliger des pertes substantielles aux vergers de mangues au Burkina Faso. Cette étude préliminaire révèle d'une importance capitale, au regard des informations fournies sur son importance en fonction des zones de production, des variétés commerciales et de ses causes probables. En effet, la présence de la maladie dans toutes les provinces prospectées, sur toutes les variétés et accessions cultivées de tout âge témoignent de la ménace sérieuse de cette maladie. Les manguiers infectés ont présenté diverses manifestations. Les flétrissements de feuilles qui évoluent progressivement aux dessèchements de rameaux des 

des champignons associés à la maladie au Burkina Faso.

branches et de l'arbre tout en entier ont été fréquemment observés dans les vergers de production. Les exsudats gommeux, des colorations vasculaires et des pourritures racinaires ont été occasionnellement constatés dans certains vergers sévèrement attaqués dans le Nahouri et le Houet. Ces symptômes sont caractéristiques du dessèchement du manguier (Ramos et al., 1997; Ploetz., 2003; Al-Adawi et al., 2006; Masood et al., 2010; Ismail., 2011). L'ensemble de tous ces symptômes peuvent se produire seul ou en association de deux ou de plusieurs dans un verger et provoqués le dessèchement des manguiers (lqbal et al., 2007; Naqvi et al., 2014).

Incidence et Sévérité du dessèchement au Burkina Faso: L'incidence et la sévérité de la maladie varient selon les provinces. La Léraba a enregistré la plus faible incidence et la plus faible sévérité. Cela, pourrait se traduire par les conditions pédoclimatiques rencontrées. En effet, cette province a reçu pour l'année 2015 une pluviométrie moyenne de $1172,525 \mathrm{~mm}$. Elle figure parmi les provinces les plus arrosées du Burkina Faso et les sols sont d'assez bonne qualité offrant ainsi des conditions favorables à l'éclosion d'un couvert végétal (www.regions.bf//MG/pdf/cascades.pdf). En revanche, la forte incidence et de sévérité moyenne ont été observées dans le Nahouri. Cette province a été moins arrosée avec une pluviométrie moyenne de $955,42 \mathrm{~mm}$ en 2015 . La faible pluviométrie serait en partie responsable des forts taux de la maladie constatés en 2016 dans le Nahouri. En effet, les symptômes du dessèchement du manguier étaient plus sévères dans les régions à plus faible pluviométrie comparativement aux plus arrosées (Khanzada et al., 2004). Selon, Moussa et al., (2011) le dépérissement est dû à une conjugaison de facteurs. La baisse de la nappe phréatique provoque une malnutrition généralisée du manguier et l'expose à la maladie. En outre les températures moyennes annuelles ainsi que la variabilité des précipitations mensuelles contribuent plus au potentiel distribution de la maladie (McDowell et al., 2014 ; Galdino et al., 2016). La province du Nahouri présente d'une manière générale des sols du type ferrugineux tropical (Felix., 1989) qui constituent $85 \%$ des sols du Burkina Faso. Ils sont généralement pauvres en matière organique, azote, phosphore et potassium. Ces sols renferment également de nombreuses autres contraintes telles que : la faible réserve en eau utile, une structure peu favorable à la pénétration racinaire, des valeurs de $\mathrm{pH}$ souvent inadéquates pour les cultures (Pallo et Thiombiano., 1989). Au Pakistan, les plus fortes incidences du dessèchement du manguier ont été enregistrées dans les zones présentant des sols peu profond, compact à texture sablonneuse ou limoneuse, avec des pH 8.5-9.0 (Naqvi et Perveen, 2015). En outre, une variabilité de la manifestation de la maladie a été constatée en fonction des sources d'irrigation, de certaines pratiques agricoles telles que l'utilisation $d u$ NPK sans recommandation, les cultures intercalaires (cultures fourragères) dans les vergers et l'entretien des manguiers à travers la taille des arbres.

Prévalence du dessèchement sur les variétés commerciales: L'analyse de la variance des prévalences moyennes de la maladie montre une différence significative entre les cinq variétés les plus commercialisées du Burkina Faso. La variété Amélie a enregistré l'incidence moyenne la plus élevée tandis que Brooks a obtenu la plus faible valeur moyenne. Des études ont révélé également la variabilité de cette maladie sur certaines variétés de manguier provenant des districts de Tando Qaiser, Hyderabad, Sindh, et de Multan au Pakistan (Khaskheli et al., 2011; Naqvi et Perveen., 2015). En effet, Naqvi et Perveen.,(2015) ont constaté la plus forte incidence de cette maladie sur la variété Chaunsa (32\%) et le plus faible taux $(0.83 \%)$ chez Almas. Selon ces auteurs, cela pourrait se traduire par un comportement variétal. Certaines variétés auraient la capacité de surmonter cette maladie durant un certain âge de leur évolution lié à leur résistance naturelle. En outre, les besoins mensuels en eau des manguiers peuvent atteindre 200 à $250 \mathrm{~mm}$ pendant la saison la plus chaude et la plus sèche (PIP., 2013). Ainsi, le stress hydrique et les fortes températures généralement observés entre Mars et Avril au Burkina Faso pourraient affaiblir les manguiers et entrainer des fortes incidences de la maladie surtout au niveau des variétés précoces comme Amélie.

Inventaire et taux d'infection des différentes espèces de champignon : Les isolements réalisés à partir des échantillons malades ont permis d'inventorier sept espèces de champignons. II s'agit notamment de $: P$. microspora, B. theobromae, Fusarium sp, Curvularia sp, C. gloesporiodes, $B$. cinerae et $A$. niger. Cette complexité des espèces de champignons associées au dessèchement du manguier a été également signalée par divers auteurs. En effet, trois (03) espèces à savoir Botryosphaeria ribis, Hendersonulla toruluoides et Macrophoma mangifera ont été détectées au Mali (Moussa et al., 2011), quatre (04) espèces de champignons dont Lasiodiplodia theobromae, Hendersonulla toruluoides, Fusarium sp et Phoma sp au Niger (Adamou., 2005). Au Pakistan, Khanzada et al., (2004) ont pu inventorier 11 espèces dont $A$ niger, Curvularia lunata, Drecshslera australiensis, Fusarium solani, F. semitectum, L. theobromae, Pythium 
aphanidermatum, Rhizoctonia solani, Rhizopus oryzae, Trichoderma harzianum et $T$. longibrachiatum avec $L$. theobromae la plus abondante suivie de F. solani en terme de fréquence. Leghari., (2005) a isolé 12 espèces de champignons appartenant à 10 genres différents à partir de manguiers présentant l'ensemble des symptômes du dessèchement du manguier. Parmi les huit espèces de champignon observées au Burkina Faso $P$. microspora, B. theobromae, et Fusarium sp ont été fréquemment rencontrées sur tous les organes et dans toutes les provinces. En effet, ces trois espèces ont été citées comme responsables du dessèchement de certaines espèces de plantes. Au Brésil, en Oman et au Pakistan deux espèces de champignon à savoir Ceratocystsis fimbriata Ellis \& Halst, et $L$ theobromae (Pat.) Griffon \& Maubl ont été isolées à partir des manguiers souffrant de cette maladie (Galdino et al., 2016; Saeed et al., 2011; Oliveira et al., 2015). En outre,

\section{CONCLUSION}

L'étude a permis d'apprécier l'importance de la maladie à travers l'evaluation de l'incidence et de la sévérité en fonction des principales zones de productions et des variétés commerciales du Burkina Faso. En outre, elle a permis d'inventorier les différentes espèces de champignons associées au dessèchement du manguier. Par ailleurs, elle a permis de révéler sur la base des caractéristiques morphologiques la présence l'espèce de champignon $B$. theobromae, connue dans de nombreux

\section{RÉMERCIEMENT}

Les auteurs de ce présent article adressent leur sincère remerciement au Centre National de Spécialisation en Fruit et Légume (CNS-FL) de l'Institut de l'Environnement et de Récherche Agricole (INERA) Farako-ba du Burkina

\section{BIBLIOGRAPHIE}

Adamou H., 2005. Déperissement des manguiers INRAN/CERRA Kollo, 1p.

Al Adawi A.O. Deadman M. L., Al Rawahi A. K., Al Maqbali Y. M., Al Jahwari A. A., Al Saadi B. A., Al Amri I. S. and Wingfield M. J., 2006. Aetiology and causal agents of mango sudden decline disease in the Sultanate of Oman, European Journal of Plant Pathology, 247-254.

AIAdawi A.O., Deadman.M.L., AIRawahi.A.K., Khan.A.J. and AlMaqbali .Y.M., 2003. Diplodia theobromae associated with sudden decline of mango in the Sultanate Of Oman. Plant Pathol. 52, 419.

Anwar A.S., Mckenry M.V. et Ahmad H.A., 2012.
$B$. theobromae a été signalée comme responsable du dépérissement du manguier en Egypte, en Australie, aux Emira Arabes Unies, et au Pérou (Sakalidis et al., 2011; Ismail et al., 2012 ; Saeed et al., 2017 ; Rodríguez-gálvez et al., 2017). Selon Fateh et al., (2016) quatre espèces de champignons à savoir Ceratocystis $\mathrm{sp}, \mathrm{L}$. theobromae, Nattrassia mangiferae et Fusarium sp sont fréquemment citées sur le dessèchement des plantes fruitières, forestières et ornementales. En effet ces espèces sont susceptibles de provoquer la mort du manguier ou des symptômes caractéristiques de la maladie suite à des inoculations. Aussi, des études conduites par Djeugap et al., (2009) ; Zhang et al., (2010) ont révélé que $P$. microspora était non seulement responsable du dépérissement mais également des maladies de taches foliaires et les pourritures des fruits de nombreuses espèces de plantes dans le monde.

pays comme responsable de la maladie. Par conséquent des études de diversité génétique et phénotypique des champignons permettront de comprendre ceuximpliqués potentiellement dans le dessèchement. Au regard de l'importance de la filière mangue pour le pays, des stratégies intégrées de lutte contre cette maladie sont également impératives pour sécuriser sa production et surtout éviter l'extinction de ce précieux fruit.

Faso et le Fonds Nationale de la Recherche et de I'Innovation pour le Développement (FONRID) pour la réalisation de cette étude.

Nematode and Fungal Communities Associated With Mango Decline of Southern Punjab. Pakistan J. Zool. 44(4), 915-922.

APROMAB, 2016. Rapport de synthèse de l'atelier bilan national de la campagne mangue, $28 \mathrm{p}$.

Bakouan B.B., 2015. Évaluation de l'efficacité du Great Fruit Fly Bait (GFFB) contre les mouches des fruits dans l'Ouest du Burkina Faso, Mémoire de fin de cycle, présenté en vue de l'obtention du diplôme d'ingénieur d'Agriculture!; Burkina Faso, Centre Agricole Polyvalent de Matourkou (CAP/M), $71 \mathrm{p}$.

Bonfigliol A., 2010. Developpement Local, Institution et Changement Climatique au Burkina Fasol: 
Analyse de la situation et recommandations opérationnelles, 90.

Cooke B.M., 2006. Disease assessment and yield loss : 43-80. In : Cooke, B.M., Jones, D.G., Kaye, B. (eds), Epidemiol. plant Dis. Springer, Netherlands.

Djeugap F.J., Fontem D.A. et Kuiate J.R., 2009. État sanitaire post-récolte de la mangue commercialisée dans la ville de Dschang et efficacité in vitro des huiles essentielles contre Colletotrichum gloeosporioides Penz., agent causal de l'anthracnose. Commun. l'Association Française Prot. des Plantes 9e CIMA 77-91.

Fateh F.S., Kazmi M.R., Ahmad I. et Rashid A., 2016. Role of different fungi for developpement of decline symptoms in citrus, mango and guava. Sci.Int. (Lahore) 28(2), 1263-1267.

Felix P.I., 1989. Perspectives d'amenagement pastoral dans la province du Nahouri-Pô Burkina Faso mémoire Université Cheikh Anta Diop de Dakar, 53.

Galdino, TarcísioVisitin da Silv., Kumar, Sunil Oliveira., Leonardo .S.S., Alfenas.C.Neven., Lisa G.Alsadi., Abdullah .M.Picanço., Marcelo C., 2016. Mapping Global Potential Risk of Mango Sudden Decline Disease Caused by Ceratocystis fimbriata. PLoS One 11(7), 1-18.

Guira M., 2003. Rapport d'activité campagne agricole, INERA, Programme CMFPT/ Burkina Faso, 13.

Hassan Z.U. et Nazami N., 2017. Potential risk to mango orchards: mango sudden decline caused by Ceratocystis fimbriata. Pak. J. Phytopathol., 29(1), 181-185.

Iqbal Z., Ehsan E.V., Muhammad.S., Kafeel.A., Zafar.I.K., Malik M. Tariq and Muhammad.D. 2007. Determination of different decline disorders in mango orchards of the Punjab, Pakistan. Pakistan J. Bot. 39(4), 1313-1318.

Ismail A.M., 2011. Studies on the fungal diseases of mango with particular reference to diseases caused by botrosphaeria species thesis of University of catania.

Ismail A.M., Cirvilleri G., Polizzi .G., Crous P. W.,Groenewald J. Z., Lombard.L., 2012. Lasiodiplodia species associated with dieback disease of mango (Mangifera indica) in Egypt. Australas. Plant Pathol. 41(6), 649-660.

Kazmi M.R., Fateh.F. S., Majeed.K., Kashkhely. M.A., Hussain.I., Ahmad A., Jabeen.2005., Incidence and etiology of mango sudden death phenomenon in Pakistan. Pak. J. Phytopathol., 17((2)), 154-158.

Khanzada A.L.I.M., Lodhi M.A. et Shahzad S., 2004. Pathogenicity of Lasiodiplodia theobromae and Fusarium solani on mango. Pak. J. Bot., 36(1), 181-189.

Khaskheli M.I., Jiskani M. M., Soomro M. H., Talpur M. A. and Poussio G. B., 2011. Prevalence of Mango Sudden Decline / Death Syndrome ( Msds ) on Various Varieties At the Orchards of Different Age in the Vicinity of Tando Qaiser, Hyderabad, Sindh. Pak. J. Agri., Agril. Engg., Vet. Sci., 27(2), 160-167.

Larignon B et Dubos P., 1997. Fungi associated with Esca disease in grapevine. Eur. J. Phytopathol. 103, 147-157.

Leghari T.N., 2005. Epidemiology and yield losses by sudden death syndrome in mango orchards of Sindh and its possible control. M. Sc. Thesis. Sindh Agriculture University, Tandojam, Pakistan.

Masood, A. Shafqat, S. Asif, M. et Malik S.N.H.., 2012. Role of nutrients in management of mango sudden death disease in Punjab, Pakistan. Pak. J. Zool. 44(3), 675-83.

Masood A., Shafqat.S., Naeem.I., Muhammad.T.M., and Munawer.R. K., 2010. Methodology for the evaluation of symptoms severity of mango sudden death syndrome in Pakistan. Pak. J. Bot., 42(2), 1289-1299.

McDowell W., Benson A. et Byers J., 2014. Climate controls the distribution of a widespread invasive species: implications for future range expansion. Freshw. Biol. 4(59).

Moussa N., Michiel A. et Salif K., 2011. Manuel sur le dessèchement des manguiers dans la région de Koulikoro, 46.

Naqvi A.H.S. et Perveen R., 2015. Mango quick decline manifestation on various cultivars at plants of particular age in the Vicinity of district Multan. Pak. J. Phytopathol. 27(1), 31-39.

Syed.A.H.N., Rashida.P., Tariq Malik. M., Owais Malik., Ummad ud Din Umer., Sajid Wazeer.M., Ateeq ur Rehman., Taha, Majid.,Zaighum, Abbas., 2014. Characterization of symptoms severity on various mango cultivars to quick decline of mango in district Multan. Int. J. Biosci. | IJB | 4(11), 157-163.

Oliveira.L.S.S., Harrington.T.C.,Ferreira.A.,Damacena.M.B., AlSadi. A. M., Al-Mahmooli.I. H. S., and Alfenas. 
A.C., 2015. Species or Genotypes? Reassessment of Four Recently Described Species of the Ceratocystis Wilt Pathogen, Ceratocystis fimbriata, on Mangifera indica. Phytopathology 105(9), 12291244.

PAFASP, 2011. Cartographie pilote des vergers de manguiers au Burkina Faso, 76p.

Pallo F.J.P. et Thiombiano L., 1989. Les sols ferrugineux tropicaux lessivés a concretions du Burkina Faso caracteristiques et contraintes pour I'utilisation agricole Bunasols BP 7142 Ouagadougou Burkina Faso, 21p.

PIP, 2013. Itinéraire technique mangue (mangifera indica), 88p.

Ploetz, R.C. David, B. and Schaffer B., 1996. A reexamination of mango decline in Florida. Plant Dis. 80(6), 664-668.

Ploetz R.C., 2003. Diseases of mango. Dis. Trop. Fruit Crop. Ploetz R.C., Ed., APS Press St. Paul; MN; USA. 327-363.

Pruvost.O.,Boyer.C., Vital.K.,Verniere.C., Gagnevin.L., Somda.I., 2011. First Report in Burkina Faso of Xanthomonas citri pv. mangiferaeindicae Causing Bacterial Canker on Mangifera indica. Plant Dis. 95, 1312.

Ramos .L.J., Davenport .T.L., McMillan .Jr. R.T. et Lara .S.P., 1997. The resistance of mango (Mangifera indica) cultivars to tip dieback disease in Florida. Plant Dis. 81(5), 509-514.

Rehman, Abdul., Abbas, asawar., Khan, Ahmad.Khan. and Mehboob S., 2011. Investigations on mango sudden death syndrome affected plant parts in district Muzaffargarh. Pak. J. Phytopathol. 23(2), 125-130.

Rodríguez-Gálvez., Edgar, Guerrero., Pakita, Barradas., Carla, Pedro Crous., Artur, Alves., 2017. Phylogeny and pathogenicity of Lasiodiplodia species associated with dieback of mango in Peru. Fungal Biol. 121(4), 452-465.

Saeed.E.E.,Sham.A., Ayah.A., Khawla .A. A., Tahra.S.A., Rabah.I.,Khaled.E.,AbuQamar, Synan.F., 2017. Detection and Management of Mango Dieback Disease in the United Arab Emirates. Int. J. Mol. Sci. 18(10), 2086.

Saeed S., Khan M.I. et Masood A., 2011. Symptom development after artificial inoculation of Botryodiplodia theobromae, a possible causal organism to quick decline in mango trees. Pakistan J. Agric. Sci. 48(4), 289294.

Sakalidis.M.L.,Ray.Jane.D.,Lanoiselet.V., Hardy.G. E.St.J., Burgess.T. I.,2011. Pathogenic
Botryosphaeriaceae associated with Mangifera indica in the Kimberley Region of Western Australia. Eur. J. Plant Pathol. 130(3), 379-391.

Suresh V., 2014. Studies on mango gummosis with special reference to Lasiodiplodia theobromae ( Pat .) Griffon \& Moube Thesis submitted to the Acharya N.G. ranga agricultural university.

Zhang M., Wu H., Tsukiboshi T. et Okabe I., 2010. First report of Pestalotiopsis microspora causing leaf spot of hidcote (Hypericum patulum) in Japan. Plant Dis. 94(8), 1064.

Zombré C., 2016. Diversité génétique et pathologique de Xanthomonas citri pv. mangiferaeindicae, bactérie responsable de la maladie des taches noires de manguier au Burkina Faso et en Afrique de l'Ouest. Thèse de Doctorat Unique, Université Ouaga 1 Pr Joseph KI-ZERBO.

www.regions.bf//MG/pdf/cascades.pdf consulté 02 Mars 2018. 